\title{
Magnetostatic interaction fields in first-order-reversal-curve diagrams
}

\author{
Adrian Muxworthy a) and Wyn Williams \\ Institute of Earth Science, University of Edinburgh, Kings Buildings, West Mains Road, Edinburgh, \\ EH9 3JW, United Kingdom
}

(Received 12 July 2004; accepted 19 December 2004; published online 7 March 2005)

\begin{abstract}
The contribution of magnetostatic interaction fields in magnetic systems during first-orderreversal-curve (FORC) simulations has been systematically addressed using a dynamic micromagnetic algorithm. The interaction field distributions (IFD) display a nonlinear dependency on the field history and intergrain spacing, and are commonly asymmetric. The IFDs tend to be more Gaussian on average than Cauchian as predicted analytically for disordered systems, due to ordering during FORC diagram determination. The spreading of the FORC distribution in the vertical direction of the FORC diagram is shown to be directly related to the mean standard deviation of the IFD during the FORC measurement, with a small offset related to the smoothing factor. (C) 2005 American Institute of Physics. [DOI: 10.1063/1.1861518]
\end{abstract}

\section{INTRODUCTION}

In recent years first-order-reversal-curve (FORC) diagrams have been proposed as an experimental method for the characterization of ferromagnetic systems. ${ }^{1,2}$ The FORC method originates from the well-studied phenomenological Preisach $^{3}$ theory of hysteresis. However, as the FORC diagram is defined from a purely experimental procedure it has certain features that are unique to it. This has led for a need to understand the origin of these observed features from a theoretical point of view.

Constructing a FORC diagram requires lengthy measurements, which has only recently become possible with modern instrumentation. Each FORC is measured by saturating the sample, decreasing the field to a value $H_{A}$, and reversing the field sweep to the saturated state in a series of field steps $\left(H_{B}\right)$. This process is repeated for many values of $H_{A}$. The magnetization $M\left(H_{A}, H_{B}\right)$ is measured at each step and the mixed second derivative taken to give the FORC distribution, ${ }^{2}$

$$
\rho\left(H_{A}, H_{B}\right)=-\partial^{2} M\left(H_{A}, H_{B}\right) / \partial H_{A} \partial H_{B} .
$$

To construct the FORC diagram, a quadratic surface is fitted over a local area defined by the smoothing factor (SF). The larger SF, the greater the number of points used. These surfaces are combined to give a piecewise quadratic surface. When the distribution is plotted as a contour plot of $\rho\left(H_{A}, H_{B}\right)$, i.e., a FORC diagram, it is convenient to rotate axes by changing coordinates from $\left\{H_{A}, H_{B}\right\}$ to $\left\{H_{C}=\left(H_{B}\right.\right.$ $\left.\left.-H_{A}\right) / 2, H_{U}=\left(H_{B}+H_{A}\right) / 2\right\}$.

The FORC diagram and the Preisach distribution will be identical for a system correctly described by the classical Preisach model (CPM). However, as many samples are not properly described by the CPM, then this leads to unusual features in the FORC diagram such as negative regions and asymmetry.

\footnotetext{
a) Author to whom correspondence should be addressed; present address: University of Southampton, Southampton Oceanography Centre, European Way, Southampton, SO14 3ZH, UK; FAX:+44 23 80593059; electronic mail: adrian.muxworthy@soton.ac.uk
}

Magnetostatic interactions are known to be one physical mechanism, which can generate non-CPM behavior. For example, another mechanism is multidomain behavior. Previous models ${ }^{1,4,5}$ have shed some light on the effect of interactions on the FORC diagram, e.g., broadening of the FORC distribution in the $H_{U}$ directions and enhancement of negative regions within the lower half of the FORC diagram. However, the shape and behavior of the interaction field have not been systematically addressed. Here we extend these studies to further examine, using a micromagnetic model, the role of interactions in single-domain (SD) assemblages and the behavior of the interaction field during FORC measurement.

Previous work on interaction fields has shown that for a disordered system of dipoles the local interaction field for volume concentrations $p<15-20 \%$ has a Cauchy distribution, while for higher concentrations the distribution is Gaussian. ${ }^{6,7}$ However, during a FORC diagram measurement, the distribution is never disordered due to the highly asymmetric measuring algorithm. The interaction field distribution (IFD) of such ordered magnetic systems is often asymmetric and the arithmetic variance of the IFD as a function of the magnetization. ${ }^{8-11}$

\section{THE MICROMAGNETIC MODEL}

The three-dimensional micromagnetic algorithm used in this study is a combination of a minimum energy conjugategradient (CG) algorithm and a dynamic algorithm which solves the Landau-Lifshitz-Gilbert (LLG) equation. ${ }^{12}$ The reasoning behind this approach is that the dynamic algorithm gives the more rigorous solution since the magnetization between stable states must follow a physically reasonable path dictated by the LLG equation of motion, however, it is relatively slow (two orders of magnitude slower) compared to the CG method. In this combination algorithm, we use the CG algorithm to rapidly generate an initial guess for the magnetic structure, which is then put into the dynamic solver. This increases the efficiency of the algorithm by roughly an order of magnitude compared to the dynamic 


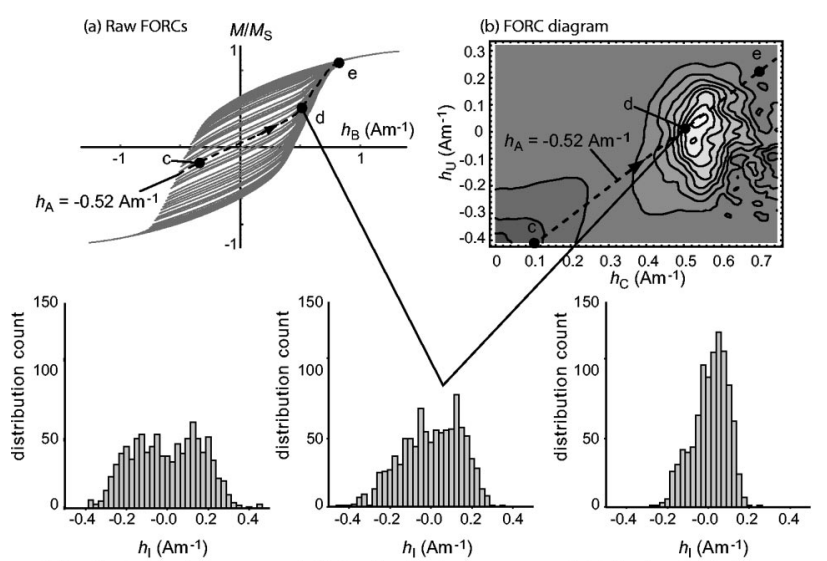

(c) distribution for $h_{\mathrm{B}}=-0.34 \mathrm{Am}^{-1}$ (d) distribution for $h_{\mathrm{B}}=0.52 \mathrm{Am}^{-1}$ (e) distribution for $h_{\mathrm{B}}=0.91 \mathrm{Am}^{-}$

FIG. 1. Raw FORCs (a) and FORC diagram (b) for an assemblage of 1000 ideal SD grains with randomly orientated uniaxial anisotropy with grain separation $d=1.3$. In (c), (d), and (e) the parallel interaction field distribution histogram is shown for three selected $h_{B}$ values for the FORC with $h_{A}$ $=-0.52 \mathrm{~A} \mathrm{~m}^{-1}$ (a). The selected FORC is also depicted on (b). In (b) a SF of 3 was used to fit the FORC distribution.

solver alone, and was found to produce the same hysteresis behavior. The combined method is more robust than the CG method alone, as it minimizes the torque on each discretized magnetic moment compared to the CG method, which only minimizes the total energy. The dynamic solver produces lower-energy states than the CG algorithm alone. We use fast Fourier transforms (FFT) to calculate the demagnetizing energy which allows the high resolution needed to examine arrays of interacting grains. This algorithm differs from that used in Muxworthy et al., ${ }^{4,13}$ who used only the CG algorithm.

We model three-dimensional arrays of $10 \times 10 \times 10 \mathrm{SD}$ cubic grains distributed evenly. Initially we consider assemblages with identical uniaxial anisotropy constant $K$, then assemblages with lognormal distributions of uniaxial anisotropy constant $K$. In both cases the easy axis of the anisotropy is randomly orientated for each grain. We examine the interaction field through each simulation. As with our previous study, ${ }^{4}$ we concentrate on a relatively soft magnetic material magnetite $\left(\mathrm{Fe}_{3} \mathrm{O}_{4}\right)$, which has a reduced anisotropy $K$ of $\sim 0.1 K_{d}$ (where $K_{d}=\mu_{0} M_{S}^{2} / 2: \mu_{0}$ is the permeability of free space and $M_{S}$ the spontaneous magnetization). All fields are given in reduced units, i.e., $h=H / H_{R}$, where $H_{R}$ $=2 K / M_{S}$.

\section{INTERACTIONS BETWEEN IDENTICAL SD PARTICLES}

The FORC diagrams (Fig. 1) calculated using 120 simulated FORCs are essentially the same as those reported by Muxworthy et al. ${ }^{4}$ for assemblages of 1000 SD grains with uniaxial randomly distributed anisotropy using 100 FORCs. The FORCs are smoother than those reported in Muxworthy et al. ${ }^{4}$ because of the improved numerical algorithm [Fig. 1(a)]. We consider the effect of variations in interaction spacing $d$ (the distance between grains divided by grain dimension) on the magnetostatic interaction field. The IFDs are calculated directly in the micromagnetic program. At the center of each grain the interaction field vector due to other
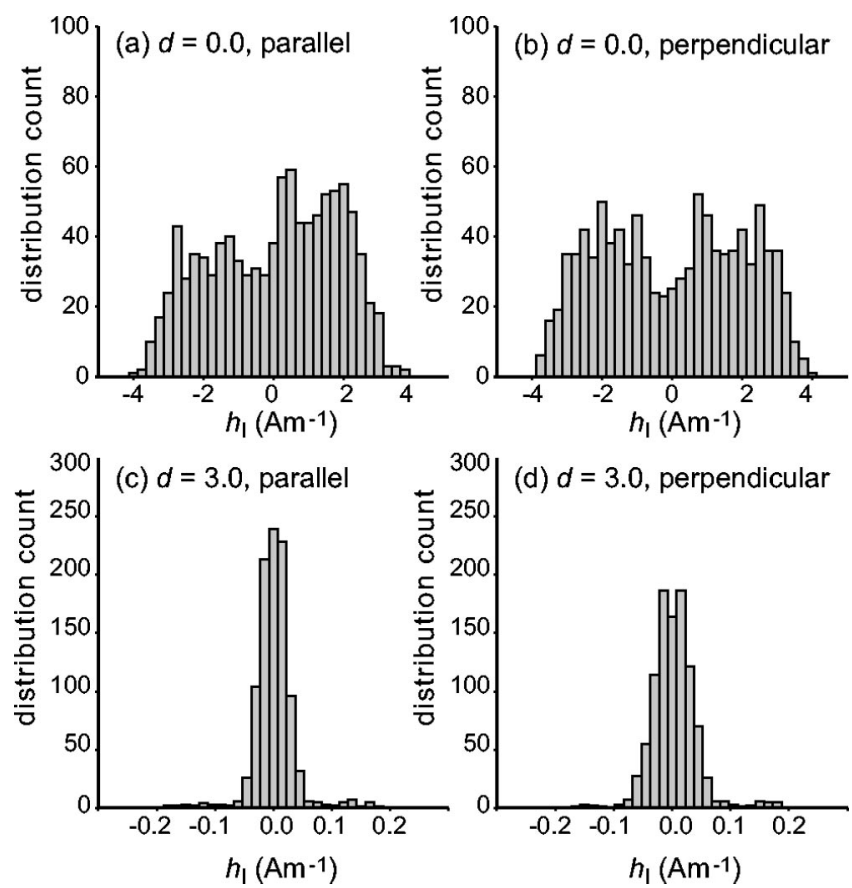

FIG. 2. Parallel and perpendicular interaction field distributions for (a) and (b) $d=0.0$, and (c) and (d) $d=3.0$, for $h_{A}=h_{B}=-0.04 \mathrm{~A} \mathrm{~m}^{-1}$. Note the different $h_{I}$ scales.

grains in the assemblage is recorded at each field step in the FORC diagram. The IFDs are calculated both parallel and perpendicular to the external field direction. The IFD was calculated for several directions in the plane normal to the field, and was found to be independent of direction.

The FORC diagram for a moderately interacting assemblage $(d=1.3)$ is shown in Fig. 1(b). The origin of the large negative region in the bottom left of the diagram has been discussed in detail previously. ${ }^{4}$ Selected IFDs for the interaction field $h_{I}$ parallel to the external field are also depicted in Fig. 1 for a single FORC, i.e., $h_{A}=-0.52 \mathrm{~A} \mathrm{~m}^{-1}$. As $h_{B}$ increases the IFD shifts towards positive values and the width or standard deviation reduces. The IFD changes shape quite markedly near the main switching field which is at $h_{B}$ $\sim 0.55 \mathrm{~A} \mathrm{~m}^{-1}$.

The IFDs in directions parallel and perpendicular to the applied field are shown for $d$ in Fig. 2 for two different assemblages, strong and weak interacting systems, i.e., $d$ $=0.0$ and $d=3.0$, respectively. As $d$ decreases the standard deviation of the distribution clearly increases. The parallel IFD is clearly asymmetric, the asymmetry being dependent on the field and magnetization [Figs. 1(c)-1(e)]. The perpendicular IFDs display greater symmetry.

The arithmetic mean of the parallel and perpendicular IFDs is plotted in Fig. 3 versus normalized magnetization for strongly and moderately interacting systems, i.e., $d=0.0$ and 1.3 , respectively. For $d=0.0$ the arithmetic mean of the parallel IFD displays an almost linear dependency on the magnetization [Fig. 3(a)] throughout the FORC measurement. For the same regime, the mean of the perpendicular field displays no strong dependency, but its absolute values are much smaller than the parallel mean values. As the interac- 

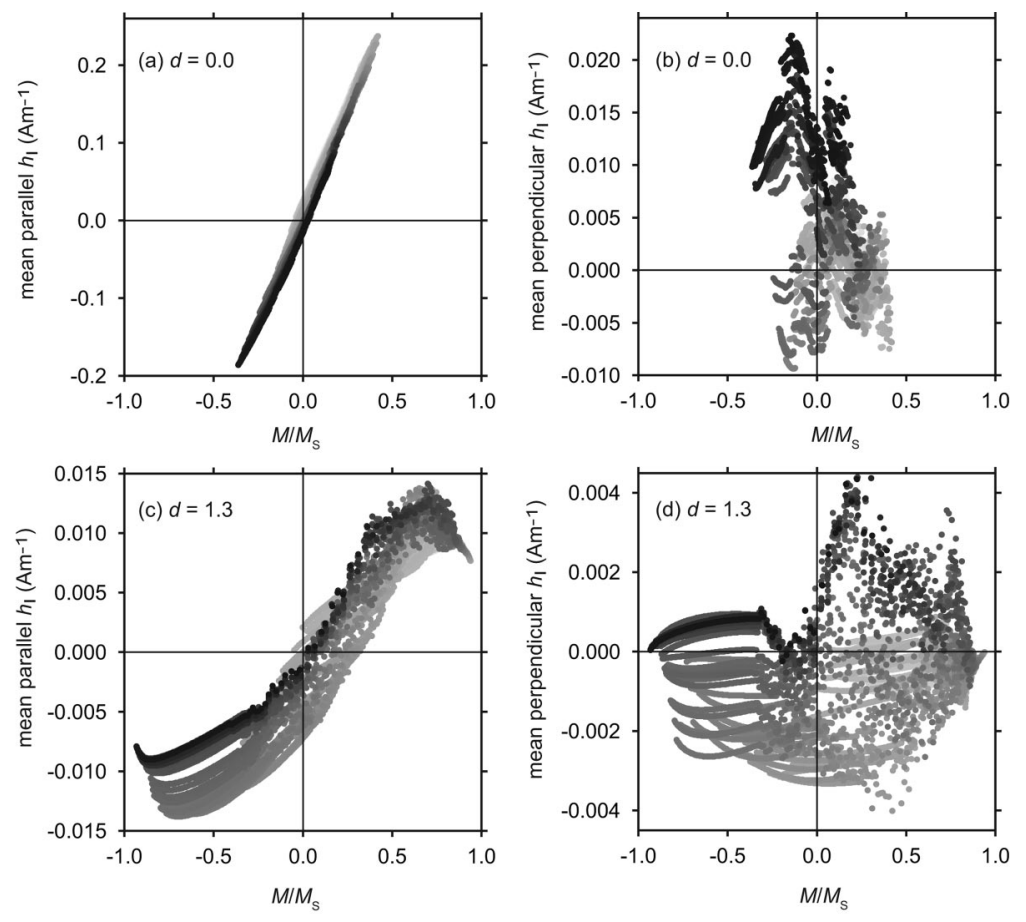

FIG. 3. Arithmetic mean parallel and perpendicular $h_{I}$ vs $M / M_{S}$ for (a) and (b) $d=0.0$, and (c) and (d) $d$ $=1.3$, for all the FORC data points in a simulation. As the FORC simulation progresses, i.e., $h_{A}$ decreasing from positive to large negative values, the shading of the points becomes darker.

tions decrease, there is a complex nonlinear dependency of both the parallel and perpendicular means on $M / M_{S}$.

For $d=0.0$, the parallel standard deviation displays a weak inverse relationship on $M / M_{S}$ [Fig. 4(a)]. As $d$ is increased the standard deviation displays a complex asymmet-
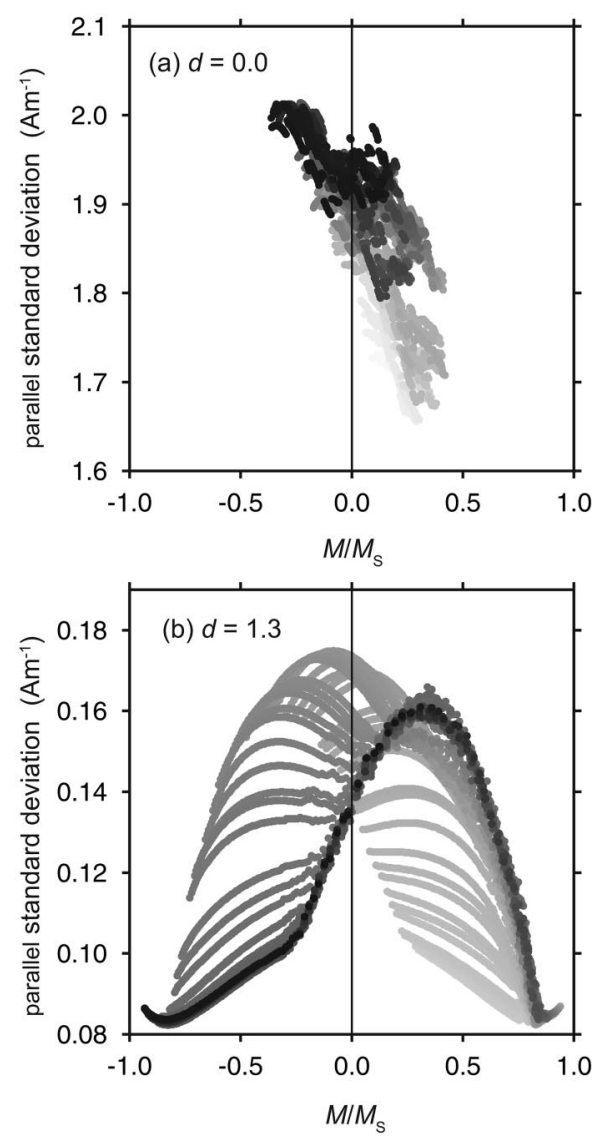

FIG. 4. Parallel arithmetic standard deviation of $h_{I}$ vs $M / M_{S}$ for (a) $d$ $=0.0$ and (b) $d=1.3$, for all the FORC data points in a simulation. ric relationship with $M / M_{S}$, which is highly dependent on $h_{A}$ [Fig. 4(b)]. The asymmetry is much more pronounced than in studies of other magnetic measurement procedures. ${ }^{8,10,11}$

In Fig. 5(a) the mean arithmetic mean of the IFD for the entire FORC measurement versus $d$ for both the parallel and perpendicular directions is shown. Similarly the mean standard deviation is shown in Fig. 5(b). Below $d \sim 1$ the average parallel arithmetic mean increases sharply with decreasing $d$ for the parallel uniform regimes. That is, for strongly interacting regimes there is a mean bias in $h_{I}$ towards the applied field direction throughout the FORC diagram derivation. The mean perpendicular arithmetic mean is almost independent of $d$. In contrast both the average parallel and perpendicular standard deviations display a strong dependency on $d$ up to $d=5$, where the standard deviation is small.

\section{INTERACTIONS BETWEEN NONIDENTICAL SD PARTICLES}

The FORC and Preisach diagrams are essentially statistical problems examining distributions of particle behavior. To make the model more representative, we vary the coercivity of the assemblage. For computation simplicity the uniaxial anisotropy value $K$ was varied rather than the grain volume, however, the net effect is similar. ${ }^{4,5}$ The coercive force distribution for powders and natural samples is commonly, though not always, lognormally distributed. The mean of the lognormal distribution was kept constant at $0.1 K_{d}$, just the width of the lognormal distribution was varied, i.e., the lognormal variance $\sigma_{l}$.

The effect of increasing the coercivity distribution width is to stretch the FORC distribution out along the $h_{C}$ axis [Fig. 6(a)], with an overall appearance closer to that of the experimental results. ${ }^{2,14,15}$ Interactions cause the FORC distribution to broaden in the $h_{U}$ direction, with preferential broadening at low $h_{U}$. This is because on average, the contribution of 

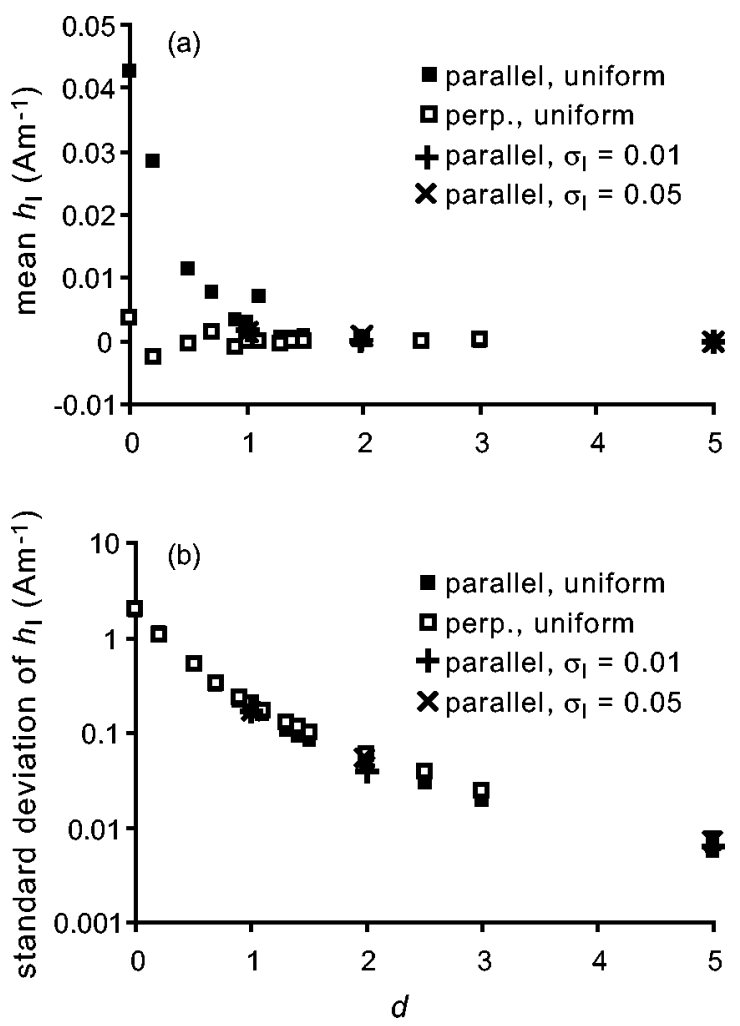

FIG. 5. (a) Average mean $h_{I}$ for the entire FORC simulation vs $d$ for uniform anisotropy (both parallel and perpendicular $h_{I}$ ) and assemblages with lognormally distributed anisotropy (only parallel $h_{I}$ ). In (b) the average standard deviation.

interactions to the assemblage's magnetic behavior is in direct competition with the anisotropy energy; the higher or harder the anisotropy, the less the influence of the interactions on assemblage behavior. ${ }^{13,16}$ This effectively means that magnetostatic interactions will cause FORC distributions to broaden more significantly at low $h_{C}$ values causing a uniform distribution to become more "pear shaped."

The IFDs become more regular in shape, though there they are still asymmetric. The mean arithmetic mean and standard deviation for the parallel IFD plot, within the same areas as the uniform distributions (Fig. 5) for $\sigma_{i}=0.01$ and 0.05 .
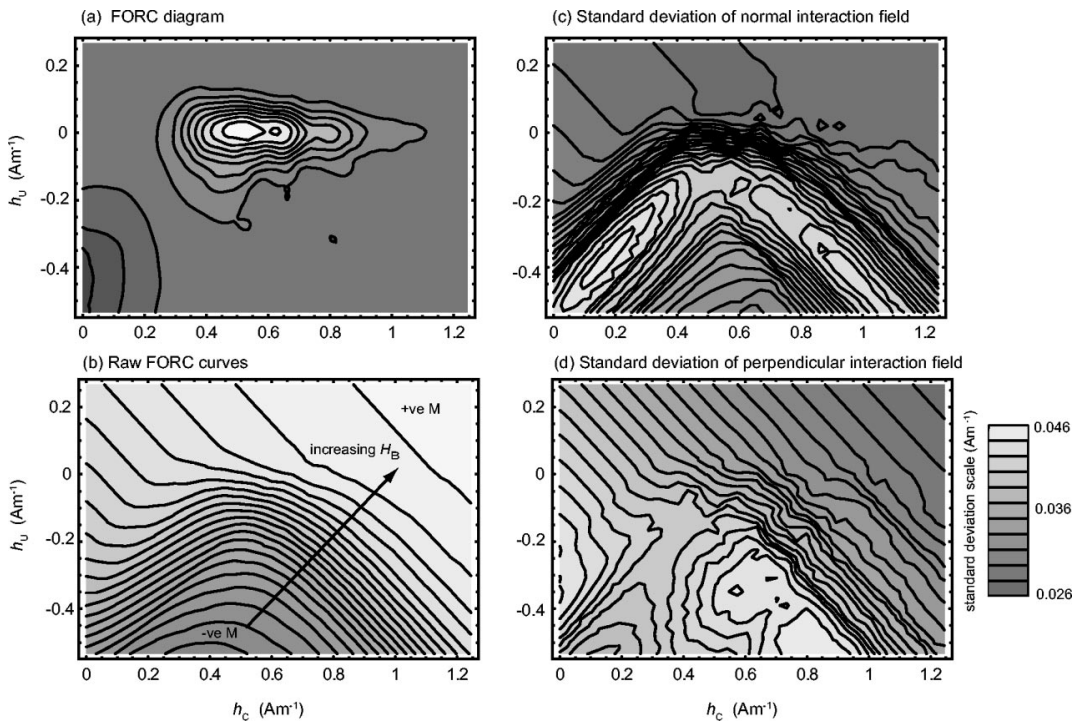

In Figs. 6(c) and 6(d) the standard deviation of the parallel $h_{I}$ and perpendicular $h_{I}$ in FORC space for the same assemblage, as shown in Fig. 6(a). In Fig. 6(b), the raw magnetization is plotted in FORC space. It is clear, that the field history (both $h_{A}$ and $h_{B}$ ) strongly affects the standard deviation of $h_{I}$. For example, on the upper part of Fig. 6(c), where $h_{A}$ is positive or has small negative values, the standard deviation is relatively uniform and narrow, but as $h_{A}$ decreases the standard deviation increases near the switching regions. The perpendicular standard deviation also shows a dependency on the switching ridges [Fig. 6(d)].

\section{DISCUSSION AND CONCLUSIONS}

The interacting field distributions within the models display strong dependencies on both field history and the spacing $d$ and orientation, in agreement with similar studies. ${ }^{9}$ As $d$ decreases the standard deviation of the IFD increases. When random spatial distributions or lognormal distributions of coercive field are examined, then the interaction effects observed for assemblages of identical grains are less pronounced and smoothed. The inclusion of lognormal coercivity distributions (Fig. 6) causes the FORC distribution to spread out along the $h_{C}$ axis in agreement with other theoretical studies. ${ }^{1,5}$

Following from Néel's ${ }^{17}$ interpretation of the Preisach diagram, Pike et al. ${ }^{1}$ argued that spreading in the $h_{U}$ direction was directly linked to the variance of the IFD. To estimate this degree of spreading we introduce the parameter full width at half maximum (FWHM), which is the full width at half maximum of the FORC distribution cut through the main peak in the $h_{U}$ direction. In Fig. 7 FWHM is plotted against the average standard deviation of $h_{I}$ in the parallel direction for two values of SF. Both values of SF display the same linear dependency on the standard deviation, offset by a finite difference related to SF. Due to the method of calculating the FORC diagram, increasing SF increases FWHM. For strongly interacting systems the FWHM becomes difficult to estimate due to the irregular profile. The statement of Pike et al. ${ }^{1}$ appears to be correct for moderately interacting systems. For strongly interacting systems the FORC diagram 


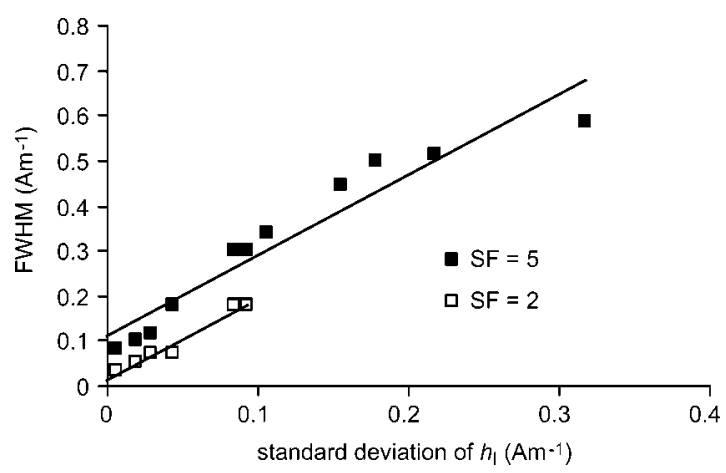

FIG. 7. Full width at half maximum (FWHM) of the FORC profiles for the uniform models vs the average standard deviation of $h_{I}$ for the entire FORC simulation. The FWHMs were calculated using SF, of both 2 and 5. The $\mathrm{SF}=2$ record is shorter due to the difficulty in estimating the FWHM for noisy distributions. Linear regression lines are depicted.

becomes irregular, while for weakly interacting systems even though the standard deviation of $h_{I} \rightarrow 0$, FWHM remains nonzero due to the FORC fitting algorithm.

It is well known that the Preisach distribution is not stable during the magnetization process of most known magnetic systems. It is possible to distinguish two kinds of modification to the Preisach distribution, the shift of the distribution in the Preisach plane and the change of IFD variance as a function of the magnetization. The former is accommodated in the moving Preisach models, and the change in the IFD variance is taken into account in the variable variance Preisach model. ${ }^{8}$ For the highly interacting systems parallel $h_{I}$ and $M / M_{S}$ are nearly linearly correlated [Fig. 3(a)], and for such cases a moving Preisach model will sufficiently describe the system. ${ }^{1}$ However, such highly interacting systems do not carry reliable magnetic signals. For more moderately and weakly interacting systems, it is necessary to include a variable variance model. For FORC diagrams it is clear from Fig. 4(b) that the variance displays nonlinear behavior, and that it would be necessary to implement an iterative algorithm to determine the IFD variance on real samples. ${ }^{8,18}$

For the distributions of nonidentical grains, the parallel IFDs were on average better described by Gaussian distributions than Cauchy distributions, even though $p$ had a maxi- mum of only $15 \%$ for $d=1$. This is in contrast to the theory of Berkov; ${ }^{7}$ however, this disagreement is probably due to the magnetic systems in a FORC measurement never being in a truly disordered state, and to a lesser extent the fixed symmetry in our model.

For highly interacting SD systems, i.e., $d<1$ or $p>0.2$, FORC diagrams do not appear to be successful at identifying the system, as the strong peaks associated with a narrow distribution of SD grains completely vanish. ${ }^{4}$ Conversely, this means that any randomly orientated sample, which displays a noticeable peak in its FORC diagram, is probably not strongly influenced by interactions.

\section{ACKNOWLEDGMENTS}

This study was funded through NERC Grant No. NER/ $\mathrm{A} / \mathrm{S} / 2001 / 00539$ to one of the authors (W.W.).

${ }^{1}$ C. R. Pike, A. P. Roberts, and K. L. Verosub, J. Appl. Phys. 85, 6660 (1999).

${ }^{2}$ A. P. Roberts, C. R. Pike, and K. L. Verosub, J. Geophys. Res. 105, 28461 (2000).

${ }^{3}$ F. Preisach, Z. Phys. 94, 277 (1935).

${ }^{4}$ A. R. Muxworthy, D. Heslop, and W. Williams, Geophys. J. Int. 158, 888 (2004)

${ }^{5}$ A. Stancu, C. R. Pike, and L. Stoleriu, J. Appl. Phys. 93, 6620 (2003).

${ }^{6}$ V. P. Shcherbakov and V. V. Shcherbakova, Izv., Acad. Sci., USSR, Phys. Solid Earth 9, 101 (1975).

${ }^{7}$ D. V. Berkov, Phys. Rev. B 53, 731 (1996)

${ }^{8}$ M. Pardavi-Horvath, E. Della Torre, and F. Vajda, IEEE Trans. Magn. 29, 3793 (1993).

${ }^{9}$ M. Cerchez, L. Stoleriu, and A. Stancu, Physica B 343, 48 (2004).

${ }^{10}$ A. Stancu, L. Stoleriu, and M. Cerchez, J. Appl. Phys. 89, 7260 (2001).

${ }^{11}$ A. Stancu, L. Stoleriu, and M. Cerchez, J. Magn. Magn. Mater. 225, 411 (2001).

${ }^{12}$ D. Suess, V. Tsiantos, T. Schrefl, J. Fldler, W. Scholz, H. Forster, R. Dittrich, and J. Miles, J. Magn. Magn. Mater. 248, 298 (2002).

${ }^{13}$ A. R. Muxworthy, W. Williams, and D. Virdee, J. Geophys. Res. 108, 2517 (2003).

${ }^{14}$ A. R. Muxworthy and D. J. Dunlop, Earth Planet. Sci. Lett. 203, 369 (2002).

${ }^{15}$ P. Postolache, M. Cerchez, L. Stoleriu, and A. Stancu, IEEE Trans. Magn. 39, 2531 (2003).

${ }^{16}$ E. Kneller, in Magnetism and Metallurgy, edited by A. Berkowitz and E. Kneller (Academic, New York, 1969), pp. 366-472.

${ }^{17}$ L. Néel, Appl. Sci. Res., Sect. B 4, 13 (1954).

${ }^{18}$ S. Garg, E. Della Torre, and E. Cardelli, J. Appl. Phys. 89, 7242 (2001). 